\title{
Dementia care in acute hospitals- A qualitative study on nurse managers' perceived challenges and solutions
}

\begin{abstract}
Aim - The aim of this study was to explore the perceived challenges of nurse managers when caring for patients with dementia in acute hospitals and identify possible solutions to address these challenges.
\end{abstract}

Background - Although dementia care in acute hospitals is suboptimal, few solutions have been identified. Multilevel factors need to be considered to promote changes in practice.

Method - Two focus groups were held with sixteen nurse managers responsible for eleven acute medical wards in Malta. Deductive qualitative analysis using an adapted version of McKinsey 7-S model was used as an a priori framework to categorise the perceived challenges and solutions.

Results - The nursing managers identified a number of organisational challenges that have a direct impact on the quality of care of patients with dementia. They also suggested a number of solutions such as realigning the hospital strategy, improving training and care coordination, redesigning the ward environment and changing leadership styles.

Conclusion - This study highlight the complexity of improving dementia care in hospitals and continues to show that a systems-wide approach is needed.

Implications for Nursing Management - Understanding the challenges and identifying possible solutions can help hospital staff provide better person-centred dementia care.

Keywords - dementia, acute care, nurse managers, focus groups, Malta 


\section{BACKGROUND}

In line with an increase in prevalence of dementia (Prince, 2015) and in view of their additional multi and co-morbidities, the number of patients with dementia admitted to acute hospitals is increasing, with one quarter of hospital beds in United Kingdom used by these individuals (Alzheimer Society, 2009). Patients with dementia have longer hospital stays, generally experience functional decline and their symptoms get worse while in hospital (Sampson et al., 2009). They are also more likely to be discharged to a care home resulting in further financial costs for local health and social care services (Connolly \& O'Shea, 2015). People living with dementia in hospitals and their family caregivers have reported negative experiences of hospital stay (Digby, Lee \& Williams, 2017). Qualitative studies have shown that acute care staff find it difficult to deliver person-centred dementia care (Clissett, Porock, Harwood \& Gladman, 2013) and meet the holistic needs of their patients (Scerri, Innes \& Scerri, 2018).

Dewing \& Dijk (2016) argued that more work needs to be done to change dementia care in hospital settings. Likewise, in a realist review of dementia-friendly interventions to improve the care of people living with dementia admitted to acute hospitals, Handley, Bunn and Goodman (2017) acknowledged that dementia training alone is not enough to improve dementia care. It is clear that there is a need for whole-systems approach for dementia care (Adams, 2008) and solutions that go beyond skill acquisition of staff (Hirst, 2017). Similarly, George, Long and Vincent (2013) recognised that there is no simple solution to improve the safety of people living with dementia in general hospitals and a multifactorial approach is necessary.

During the past decade, various solutions have been proposed to improve dementia care in acute hospitals. An initiative that consisted of the setting of dementia specialised units (Goldberg et al., 2013) showed promising outcomes, including an increase in patient's wellbeing and staff/relative satisfaction. However, Dewing and Dijk (2016) argued that there is not enough evidence as yet on their effectiveness. Such initiatives require institutional and inter-personal investment and support and their benefits may be limited to a few individuals who are admitted to these units. Considering that the prevalence of dementia in acute hospitals is all the time increasing, a multi-level approach may be required that encompasses organisational-wide changes. There is a need to identify feasible solutions that can be easily 
implemented. By exploring the perceptions of nurse managers working in hospital, it is possible to identify the challenges and potential solutions to improve the care of patients with dementia in acute wards.

\section{METHOD}

\subsection{Aim of the study}

The aim of this study was to explore what nurse managers perceive as the challenges when caring for patients with dementia in acute hospital wards and to identify possible solutions to these challenges.

\subsection{Design and sample}

The study was conducted in an acute hospital in Malta. Two focus groups were held in July 2018, with a purposive sample of invited nurse managers (consisting of charge/deputy charge nurses and senior nursing managers) coming from eleven medical wards. The decision to restrict selection from medical wards only was to enable data collection with a group with similar ward cultures. Nurse managers were selected since they should have a clear understanding of the day-to-day challenges of caring for patients with dementia in their wards and are also in work positions to identify and possibly initiate solutions. Sixteen participants (8 in each meeting) attended the two focus groups. During the focus groups, one of the researchers (AI) led the interview whilst the other (AS) observed the group interaction and made extensive notes.

The same questions (Table 1) were asked in both focus groups. These aimed at promoting discussion with the participants about their experiences of caring for patients with dementia whilst focussing on challenges and solutions. Both meetings, lasting two hours, were audiotaped and transcribed verbatim.

\section{(include Table 1)}

\subsection{Methods}

A theoretically driven approach as described by Meyer \& Ward (2014) was used to guide the design and analysis of this study. An organisational change management theory, the McKinsey 7-S Model (Watermans, Peters \& Phillips, 1980) was initially selected as the chosen theoretical 
model. According to this model, to initiate organisational change, seven different organisational factors need to be aligned and reinforced, namely: the organisational strategy, structure, systems, leadership style, skills, staff and shared values (Figure 1). The main purpose of choosing this model was that it describes the key organisational factors that influence change. Although it has been successfully used to critically analyse different challenges in hospitals (Grant, 2008; Szeto, 2017), such a model has never been applied within the context of dementia care in acute hospitals and could thus provide a new way of perceiving challenges and solutions that lead to an improvement in the quality of care for this group of patients.

\section{(insert Figure 1)}

\subsection{Data collection and analysis}

Each focus group transcript was analysed with the aid of a computer-assisted qualitative data analysis software program (NVIVO, version 11 QSR International, Victoria, Australia) to initially pre-code the data beyond the theoretical framework. This was performed to ensure that those codes that did not fall under the categories of the chosen theoretical framework were still considered (Meyer \& Ward, 2014). The second phase of analysis consisted of examining the initial codes from a conceptual and thematic perspective with a focus on perceived challenges and solutions. Following this, these themes were grouped according to McKinsey organisational factors and any theoretical and empirical gaps in the model were sought. One particular theoretical gap that was identified through the initial codes that did not fit any of McKinsey 7-S factors was related to perceived challenges associated with the physical environment of the hospital. Consequently, the physical environment or Space was included as an additional category. Thus, perceived challenges and solutions were categorised according to whether they were related to the hospital strategy, the organisational structure, the systems used, the values shared, the type of leadership styles commonly adopted, issues related to the staff and staffing levels, the skills and learning needs of the staff and the hospital environment. (Figure 2).

\section{(insert Figure 2)}

\subsection{Data quality/Rigour}

Transcriptions of the focus groups were checked against recordings on several occasions to ensure fidelity. Thematic and theoretical coding was discussed between two of the researchers (AS, AI) until agreement was reached. 


\subsection{Ethical considerations}

Ethical approval was sought and granted by the acute hospital and the University Research and Ethics Committee. Initial contact with the respective nurse managers, inviting them to attend one of the meeting, was obtained through an intermediary. Staff who attended the meetings were given an information sheet and were asked to sign an informed consent form. Pseudonyms were used when referring to the nurse managers to maintain their anonymity.

\section{RESULTS}

\subsection{Participants}

Sixteen nurse managers (nine charge/deputy charge nurses and seven senior nursing managers) who, at the time of the study, were responsible for 11 acute medical wards, participated in two focus groups ( $\mathrm{n}=8$ for each focus group). The majority of the participants were female $(81 \%)$ whilst their average age was 48 years.

\subsection{The hospital strategy}

During both focus groups, participants were of the opinion that the hospital strategy was not aimed at the care of patients with dementia, even though all acknowledged that up to half of the beds were occupied by these patients. Participants emphasised acute care over dementia care and argued that since acute hospital beds are limited, people living with dementia, especially those waiting for long-term care, have to make way for the acute cases.

Ruth: To add up to what they are saying, the environment is not so conducive to persons with dementia. The hospital was not built with the purpose to admit patients with dementia. The main aim of our hospital, which is the only public general hospital in Malta, was to admit acute patients.

Participants reported that acute care is not aligned and supported by primary and tertiary care. They felt that they were not supported enough by other institutions/departments, particularly in community and long-term care, in first reducing hospital admissions and second to facilitate care transitions back home/into the community. This was partly because community care and long-term care were not coping with the unmet needs of older persons and patients with dementia. Participants perceived that this was leading to readmissions to acute care and a prolonged length of stay in acute wards waiting for a long-term bed. 
Charlotte: Because even the discharge facilitation nurses, they tell you now she is cured from here and it is not the responsibility of the health department (anymore). Now the responsibility is passed to the elderly department but, yes, the elderly department has lack of resources and then people come back again.

One participant suggested that there was a need to sensitise the senior management by carrying out research that measures the prevalence of patients with dementia in hospital wards.

Ruth: I guess the first thing is to see at a point in time how many people with dementia we have in hospital. To see how many people there are, to see the demand. Then from that demand we can form a proposal.

Pearl: If you need something to happen at the hospital, you need numbers, you need statistics.

\subsection{The hospital structure}

Some participants, argued that the organisational structure was very rigid and heavily dominated by the medical profession, some of whom may not listen to what nurses have to say. Thus, decisions such as the use of constant watch or restraints or whether patients may require social support in the community were generally taken by the medical specialist without asking or involving the other professionals, including nurses.

Pearl: .... But even if you say there are social issues, it is not an issue (for them). And they won't listen. Some medical specialists won't listen that there are social issues.

One participant suggested that medical specialists should share responsibility and accountability with geriatric or psychiatric specialists when providing care to patients with dementia in hospital, as it was already happening in orthopaedic wards.

Margaret: But in orthopaedic (wards) we have patients who suffer from dementia and then fall. However, the approach over there is totally different from the medical wards. In the orthopaedic wards, there is the geriatric consultant who will decide upon the treatment of the patient while the orthopaedic consultant will tackle the issue related to the fracture. 


\subsection{The physical environment (Space)}

The majority of the participants argued that the hospital environment was unstable and chaotic. They felt that patients with dementia may have problems orientating themselves since no colour coding was present in the wards.

Rob: I think one of the main problems, in general, is the environment, there are no colour coding, so it is very difficult to orientate... all the doors are painted in blue, all the corridors are the same, all the bedrooms are the same, so it is very difficult to orientate for people with dementia.

Participants were also concerned that the hospital environment may be unsafe, particularly for patients with dementia who are active and seek opportunities to walk. Thus, they suggested that wards should be colour-coded and bathrooms clearly labelled. In addition, alarms, sensors (e.g. wrist bands) and low-lying beds were proposed to reduce the use of restraints and maintain patient safety.

Margaret: As we all know we can easily colour-code the wards, so that she will remember where she is going. We would be creating a room for the demented patients which will cater for them. For example, we will tell them your room is the orange coloured (room)' or whatever.

\subsection{The hospital systems}

The majority of perceived challenges mentioned by the study participants were categorised under inadequate hospital systems, ward procedures or processes such as fixed hospital routines, unnecessary patient transfers, lack of coordination between different health care professionals, wards or other departments within the hospital, odd time for discharges, lack of activities to engage patients, inadequate discharge documentation processes and ineffective application of evidence-based hospital guidelines on the use of physical restraints. Moreover, participants commented that there were no clearly defined protocols/procedures to assess patients with suspected cognitive problems, thus no differential diagnosis was being carried out.

Rob: Many are Alzheimer's and vascular dementia, but are we having these patients assessed? Is it true that they have dementia or (is it) an acute confusional state? So, we have to be clear. 
Facilitator 1: So, are you saying they are not being assessed?

Rob: I don't think that they are really being assessed ...or differentially diagnosed.

One hospital system that was mostly critiqued by the participants was the unnecessary transfers between wards.

Cynthia: We have patients transferred from the medical admissions unit or the observation ward and when the patient seems to settle in one place, he or she is moved. It is the hospital that is creating this sort of confusion.

Consequently, one of the participant suggested that patients with dementia could be fasttracked and admitted directly to a medical ward.

Rob: Another thing to improve dementia care is to avoid admissions of patients suffering with dementia to admission areas. If they do not need a monitor, for example, they must go directly to a (medical) ward to at least avoid that transition as well.

\subsection{The shared values}

Ageism and negative attitudes of nursing staff towards people living with dementia, especially those awaiting long-term care, was perceived as a concern by a number of the nurse managers. Patients were being labelled 'confused' or 'demented' or 'social case' and this led to a lack of dignity towards these patients and a lack of interest in geriatric and dementia care.

Chris: I think most of the nurses they just label the patient as 'confused'...

Jason: Recently we did an audit about what the nurses want to know more, let's say, tracheostomy care, colostomy. Dementia care: no one mentioned it.

Grace: Because there is not enough awareness... and the elderly, they are a relatively new (topic) and people do not give it importance.

Participants felt that at times hospital staff were not maintaining the patient's dignity and autonomy. This was particularly evident when deciding to use physical restraints:

Martha: Let us take the example of restraints. If the carer learned by experience that a person who is wandering has to be restrained, they do not know otherwise, so they have learned it the wrong way. You know what I mean... 
One participant suggested that to reduce ageism, staff should be trained to become more empathic towards these patients so as to change their core values.

Jason: We need to become more empathic and increase in our values and be more proactive to address certain situations, to try to reduce the gap in patients' needs.

\subsection{The leadership style}

Participants expressed their concern that the type of leadership style adopted by medical specialists and by senior management was generally autocratic and that they had minimal say in decision taking such as whether and when patients are restrained or started on enteral feeding or when discharged.

Pearl: I think they (the patients) will show us if they do not want it (the food). Then it depends upon the medical specialist; someone will say 'no we put on mittens and we keep on feeding them.' Someone will say like 'if they are refusing they are refusing, they can decide'...

Facilitator: So, the medical specialist's advice is variable.

Pearl: A lot of things is according to the medical specialist.

One participant felt that rather than using an autocratic leadership style, adopting a distributed leadership approach may be better since it enhances shared decision making.

Pearl: I don't have relievers. I have good carers and not so good carers but Ifound out that some of the good carers will even come over and tell me 'It is better if we start the intake (chart) for that patient because that patient I am observing is not eating enough'.

\subsection{Staff levels and skill mix}

All participants expressed their apprehension about the need for more staff and that staffing levels should not fluctuate from day to day. They also argued that there is a lack of supporting staff such as transport aides who would take some of the nurses' workload.

Margaret: What about the nurse:patient ratio. We have a huge issue about it. I know that most of what (the concerns) are rightly so, but the resources are what they are! 
Melanie: I agree but let me give you a different example now. We have a lot of transfers in the afternoon. How can I wash patients in the afternoon? In the afternoon, we have 10 patients to transfer.

Margaret: And that causes problems to demented patients.

Martha: And we do not have transport support.

Besides improving staffing levels, other solutions were proposed by participants, such as the recruitment of a practice nurse, the inclusion of volunteers and the identification of Dementia Champions.

Rob: What about having a practice nurse in dementia care for example. We don't have a practice nurse in dementia care.

Jason: I believe that actually Champions have to be identified among staff that have their parents passing through the experience (of caring for persons with dementia at home).

\subsection{Staff training}

All participants acknowledged that there was a skill deficit in staff related to dementia care. Table 2 lists the knowledge and skills required by hospital staff to care for patients with dementia, as identified by participants in the focus groups.

(Insert Table 2)

Continuous professional development and in-service training was suggested by almost all participants. Different sources of learning were proposed such as learning from informal carers, learning from other professionals working at other entities, learning from each other through the use of common documentation systems and learning from Dementia Champions.

\section{DISCUSSION}

This study explored the perceived challenges identified by nurse managers when caring for people living with dementia in acute hospital wards and possible solutions to overcome these 
challenges. An adapted McKinsey 7-S model (Watermans, Peters \& Phillips, 1980) was used that provided the opportunity to look at a range of organisational elements within an acute hospital in Malta and evaluate how all these elements can have a direct impact on the quality of care of patients with dementia admitted in acute hospitals. The authors of this model also argued that to achieve organisational change, all these elements need to be examined and multilevel strategies instituted (Watermans, Peters \& Phillips, 1980). Similarly, this study has shown that a multi-level approach is required to improve dementia care in hospital settings as has been advocated in other studies (Naef, Ernst, Burgi \& Petri, 2018).

Although participants were requested to focus on the challenges in caring for people with dementia in an acute hospital setting, a number of nurse managers reverted to discussing the challenges of caring for patients awaiting long-term care. This could be attributed to the fact that a significant number of older patients with cognitive impairment were in fact waiting for long-term care placement. Evidence that patients with cognitive impairment have a higher risk of being discharged from hospital and admitted to an institution is available (Fogg, Griffiths, Meredith \& Bridges, 2017). Participants in this study felt that this was due to the lack of support in the community and that hospitals were being used as an access point for entry to long-term care; which is in itself a scarce resource. This highlighted the needs for a health and social care strategy that takes an integrated approach across acute hospitals, community and long-term care settings whilst strengthening the community support as indicated by the participants in this study. Similar solutions to reduce hospital bed use by frail older persons are also well supported by clinical evidence (Philp et al., 2013). Participants indicated that patients with dementia, experience too many transitions before, during and after hospital admission. They believed that there was a need to reduce ward transitions by, for example, directing patients with dementia from the emergency department immediately to acute medical wards rather being admitted to an admission unit, thereby reducing unnecessary transfers. This reflects Fortinsky and Downs (2014) argument to optimise and reduce the burden of transitions especially in the last part of the disease trajectory.

Most of the challenges identified by the participants were related to ineffective hospital systems as a result of an organisational culture that was mainly task or staff-centred rather than person or patient-centred. Organisational culture has been found to be related to the implementation of person-centred care in hospital settings (Carlström \& Ekman, 2012) and in dementia care (Kirkley et al., 2011). The power dynamic in public hospitals has also been reported to be 
negatively associated with readiness to change (Seren \& Baykal, 2007). Similarly, participants in this study indicated that the organisational structure was rigid, medically dominated and lacked distribution of power. Nurse managers felt that more collaboration was needed especially between physicians and geriatricians. They also indicated for more democratic leadership styles to increase staff engagement. Similarly, Rokstad et al. (2015) found that nursing homes using transformational leadership styles were better in introducing personcentred dementia care practices. Whilst there are no studies relating the impact of the leadership style on person-centred dementia care in hospitals, there is clear evidence that a transformational leadership style is positively associated with staff outcomes (Cummings et al., 2010) and patient outcomes (Wong, Cummings \& Ducharme, 2013).

According to the participants, one of the most difficult challenges, was the negative attitudes of nursing staff and carers towards older adults and patients with dementia. Whilst attitudes towards older adults vary between countries (Topaz \& Doron, 2013), there is general acceptance that Western societies view older adults negatively and consider them a burden on society (Palmore, Branch \& Harris, 2005). Ageist stereotypes have also been found in acute care staff (Courtney, Tong, \& Walsh, 2000) and have been attributed to lack of training in geriatric care (Gallagher, Bennett, \& Halford, 2006). Nevertheless, person-centred care training targeting hospital staff can improve attitudes towards these patients (Surr, Smith, Crossland \& Robins, 2016). Similarly, the participants acknowledged that there was a need for more training in dementia care, especially 'empathy' training to make up for these negative attitudes which could in turn reduce the use of restraints in hospital wards.

The physical environment of the hospital was not considered to be in keeping with dementiafriendly design principles (O’Malley, Innes, \& Wiener, 2017). Participants acknowledged that the hospital setting could be disorientating for patients with dementia. Possible solutions were identified by the participants such as the use of colour coding and signage. These suggestions are similar to what have been proposed elsewhere (Leung \& Todd, 2010).

The study highlighted the complex challenges experienced by nurse managers working in acute hospital wards. As indicated in this study and elsewhere (George, Long \& Vincent, 2013), solutions are equally complex but can be achieved if a concerted effort is taken by all stakeholders, including nurse managers. The latter, especially first-line and second-line 
managers, should act as role models and catalysts in initiating discussions with dementia care experts and introduce compulsory dementia care training to new recruits together with periodical development sessions for experienced nurses. Nurse managers are in a prime position to advocate on evidence-based guidelines to carry out system changes and realign the organisational culture to an ethos where the care is truly person-centred. This study also indicates that there is a need to ensure that nurse managers are listened to by senior management and provided with all the necessary tools to guide the required organisational changes. Finally, nurse managers should monitor and evaluate the care being provided to ensure that older persons and patients with dementia are treated with dignity and respect throughout their hospital stay.

\section{LIMITATIONS}

Since this was a small-scale study in one hospital in Malta, it is not possible to generalise the findings to other hospitals, although the challenges identified in this study are similar to those in other countries (Dewing \& Dijk, 2016). Although hospital staff were informed prior to the focus groups that they would not be identified by their name, a number of participants may have hesitated from expressing their concerns for fear of exposing sub-optimal practices in their wards. Nevertheless, most of the participants' concerns were frank and felt they were being given the opportunity to vent their challenges with other colleagues who were going through similar experiences.

\section{CONCLUSION}

Achieving person-centred dementia care in acute hospital wards is complex and multiple factors need to be addressed. Nevertheless, this study identified practical solutions that nurse managers felt could improve dementia care in hospitals. Further studies are needed to identify the challenges and solutions from the perspective of other stakeholders such as physicians, hospital directors and community-service managers. Implementing solutions, however, requires whole-systems change and a realignment of these organisational elements, especially the shared values of staff, so that the care provided becomes truly person-centred. 


\section{References}

Adams, T. (2008). Nursing people with dementia and their family members-towards a whole systems approach. In Adams (Ed.), Dementia care nursing: promoting well-being in people with dementia and their families. New York: Palgrave Macmillan.

Alzheimer's Society [UK] (2009). Counting the cost. Caring for persons with dementia on hospital wards Alzheimer's Society. London. Retrieved from: http://www.alzheimers.org.uk/countingthecost

Carlström, E. D., \& Ekman, I. (2012). Organisational culture and change: implementing person-centred care. Journal of Health Organization and Management, 26(2), 175191.

Clissett, P., Porock, D., Harwood, R. H., \& Gladman, J. R. (2013). The challenges of achieving person-centred care in acute hospitals: a qualitative study of people with dementia and their families. International Journal of Nursing Studies, 50(11), 1495-1503.

Connolly, S., \& O'Shea, E. (2015). The impact of dementia on length of stay in acute hospitals in Ireland. Dementia, 14(5), 650-658.

Courtney, M., Tong, S., \& Walsh, A. (2000). Acute-care nurses' attitudes towards older patients: A literature review. International Journal of Nursing Practice, 6(2), 62-69.

Cummings, G. G., MacGregor, T., Davey, M., Lee, H., Wong, C. A., Lo, E., ... \& Stafford, E. (2010). Leadership styles and outcome patterns for the nursing workforce and work environment: a systematic review. International Journal of Nursing Studies, 47(3), 363-385.

Dewing, J., \& Dijk, S. (2016). What is the current state of care for older people with dementia in general hospitals? A literature review. Dementia, 15(1), 106-124.

Digby, R., \& Bloomer, M. J. (2014). People with dementia and the hospital environment: the view of patients and family carers. International Journal of Older People Nursing, 9(1), 34-43. 
Digby, R., Lee, S., \& Williams, A. (2017). The experience of people with dementia and nurses in hospital: an integrative review. Journal of Clinical Nursing, 26(9-10), 1152-1171

Fogg, C., Meredith, P., Bridges, J., Gould, G. P., \& Griffiths, P. (2017). The relationship between cognitive impairment, mortality and discharge characteristics in a large cohort of older adults with unscheduled admissions to an acute hospital: a retrospective observational study. Age and Ageing, 46(5), 794-801.

Fortinsky, R. H., \& Downs, M. (2014). Optimizing person-centered transitions in the dementia journey: A comparison of national dementia strategies. Health Affairs, 33(4), 566573.

Gallagher, S., Bennett, K. M., \& Halford, J. C. (2006). A comparison of acute and long-term health-care personnel's attitudes towards older adults. International Journal of Nursing Practice, 12(5), 273-279.

George, J., Long, S., \& Vincent, C. (2013). How can we keep patients with dementia safe in our acute hospitals? A review of challenges and solutions. Journal of the Royal Society of Medicine, 106(9), 355-361.

Goldberg, S. E., Bradshaw, L. E., Kearney, F. C., Russell, C., Whittamore, K. H., Foster, P. E., ... \& Porock, D. (2013). Care in specialist medical and mental health unit compared with standard care for older people with cognitive impairment admitted to general hospital: randomised controlled trial (NIHR TEAM trial). British Medical Journal, 347. doi: http://dx.doi.org/10.1136/bmj.f4132

Grant, P. (2008). 'The productive ward round': a critical analysis of organisational change. International Journal of Clinical Leadership, 16(4).

Handley, M., Bunn, F., \& Goodman, C. (2017). Dementia-friendly interventions to improve the care of people living with dementia admitted to hospitals: a realist review. British Medical Journal Open, 7(7), e015257.

Hirst, S. P. (2017). Dementia Education: Does It Change Nursing Practice? Journal of Gerontological Nursing, 43(7), 2-3. 
Kirkley, C., Bamford, C., Poole, M., Arksey, H., Hughes, J., \& Bond, J. (2011). The impact of organisational culture on the delivery of person-centred care in services providing respite care and short breaks for people with dementia. Health \& Social Care in the Community, 19(4), 438-448.

Leung, D., \& Todd, J. (2010). Dementia care in the acute district general hospital. Clinical Medicine, 10(3), 220-222.

Meyer, S., \& Ward, P. (2014). 'How to' use social theory within and throughout qualitative research in healthcare contexts. Sociology Compass, 8(5), 525-539.

Naef, R., Ernst, J., Buergi, C., \& Petry, H. (2018). Quality of acute care for persons with cognitive impairment and their families: A scoping review. International Journal of Nursing Studies, 85, 80-89.

Palmore, E. B., Branch, L., \& Harris, D. (2016). Encyclopaedia of ageism. London. Routledge.

Philp, I., Mills, K., Long, J., Thanvi, B., \& Ghosh, K. (2013). Reducing hospital bed use by frail older people: results from a systematic review of the literature. International Journal of Integrated Care, 13(4).

Prince, M. J. (2015). World Alzheimer Report 2015: the global impact of dementia: an analysis of prevalence, incidence, cost and trends. Alzheimer's Disease International. Retrieved from https://www.alz.co.uk/research/WorldAlzheimerReport2015.pdf.

Rokstad, A. M. M., Vatne, S., Engedal, K., \& Selbæk, G. (2015). The role of leadership in the implementation of person-centred care using Dementia Care Mapping: a study in three nursing homes. Journal of Nursing Management, 23(1), 15-26.

Sampson, E. L., Blanchard, M. R., Jones, L., Tookman, A., \& King, M. (2009). Dementia in the acute hospital: prospective cohort study of prevalence and mortality. The British Journal of Psychiatry, 195(1), 61-66.

Scerri, A., Scerri, C., \& Innes, A. (2018). The perceived and observed needs of patients with dementia admitted to acute medical wards. Dementia, 1471301218814383. 
Seren, S., \& Baykal, U. (2007). Relationships between change and organizational culture in hospitals. Journal of Nursing Scholarship, 39(2), 191-197.

Surr, C. A., Smith, S. J., Crossland, J., \& Robins, J. (2016). Impact of a person-centred dementia care training programme on hospital staff attitudes, role efficacy and perceptions of caring for people with dementia: A repeated measures study. International Journal of Nursing Studies, 53, 144-151

Szeto, M. C. H. (2017). 2 Challenges for a community of practice: recognising complexity with the Mckinsey $7 \mathrm{~s}$ framework. Retrieved from https://bmjleader.bmj.com/content/1/Suppl_1/A1.2.abstract

Topaz, M., \& Doron, I. (2013). Nurses' attitudes toward older patients in acute care in Israel. Online Journal of Issues in Nursing, 18(2), 46.

O’Malley, M., Innes, A., \& Wiener, J. M. (2017). Decreasing spatial disorientation in carehome settings: How psychology can guide the development of dementia friendly design guidelines. Dementia, 16(3), 315-328.

Waterman Jr, R. H., Peters, T. J., \& Phillips, J. R. (1980). Structure is not organization. Business Horizons, 23(3), 14-26.

Wong, C. A., Cummings, G. G., \& Ducharme, L. (2013). The relationship between nursing leadership and patient outcomes: a systematic review update. Journal of Nursing Management, 21(5), 709-724. 


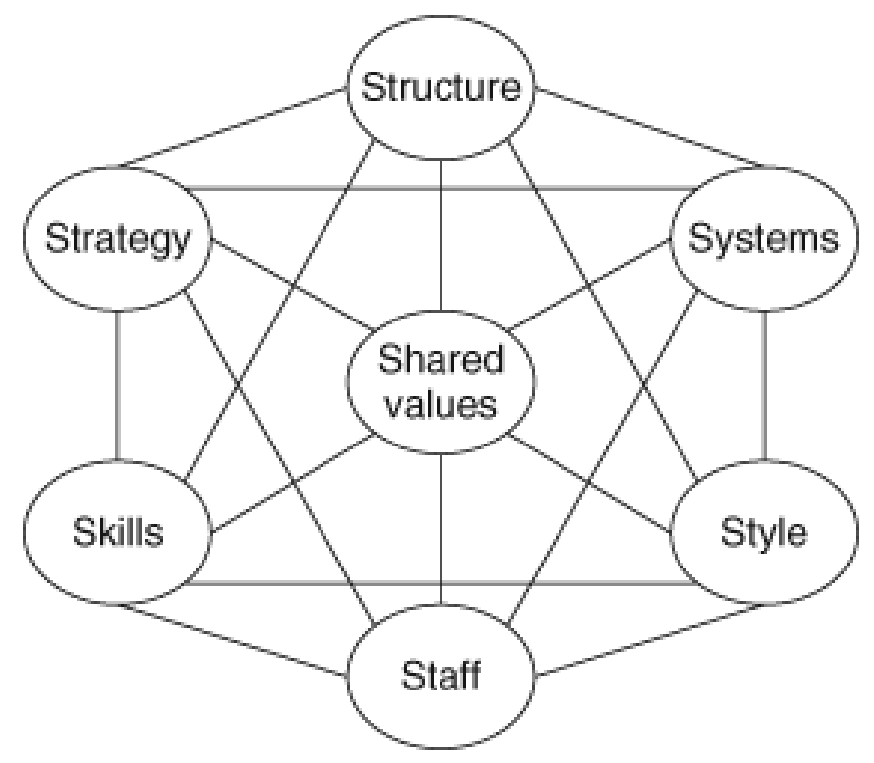

Figure 1. McKinsey McKinsey 7S model (Waterman, Peters \& Phillips, 1980 p.18)

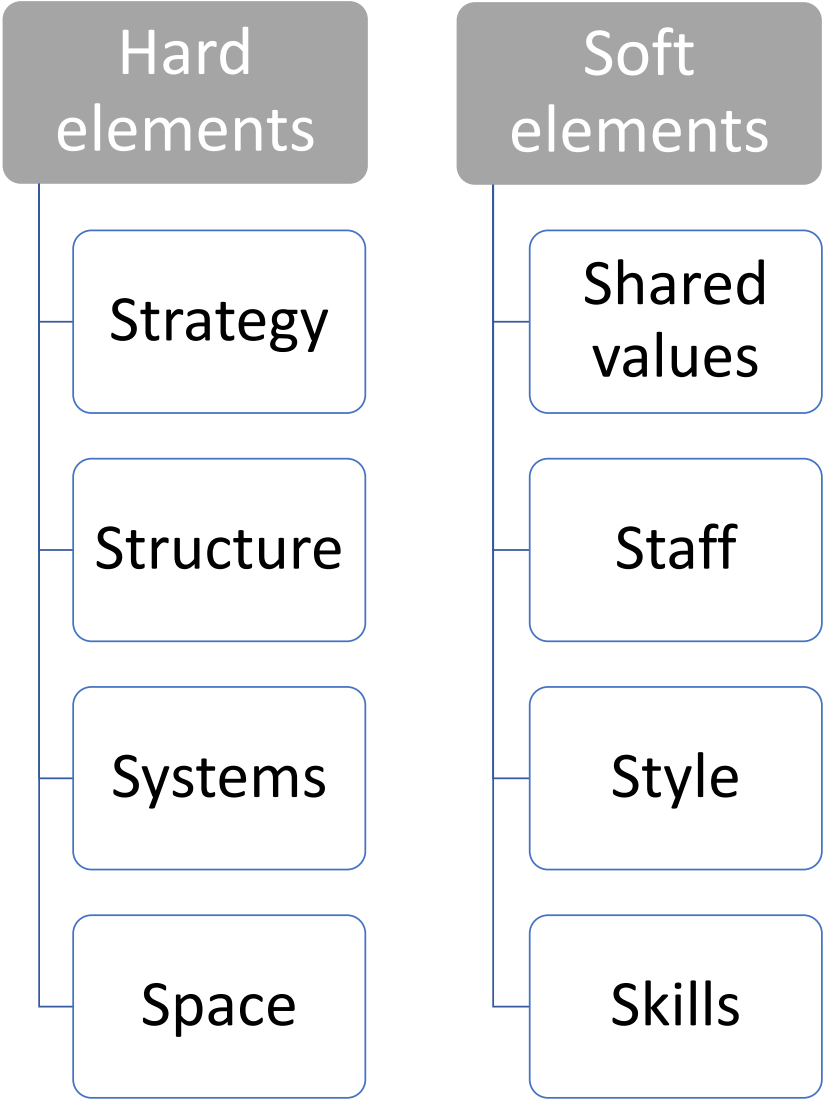

Figure 2. Hard and soft elements of the adapted McKinsey model 
1. What are the challenges you face when persons/patients with dementia are admitted in your wards?

2. What solutions/strategies can you think of to overcome these challenges?

3. What would you like to know about dementia and dementia care?

4. What would you like your staff to know about dementia and dementia care?

Table 1. Questions asked to the nurse managers during the focus groups

Understanding the life history and personality of the patient

How to deal with behavioural and psychological symptoms especially wandering

Knowing how to recognise a patient with dementia and distinguish from other conditions

Knowing how to educate and work with relatives of patients with dementia

Understanding the principles of person-centred care

How to communicate effectively with persons with dementia

How to care for older adults by assessing and managing geriatric syndromes

How to work as members of an interdisciplinary team

How to avoid excess disability

How to access community services

Knowing about the hospital guidelines on the use of restraints

How to delegate safely

Knowing about risk factors and early signs of dementia

How to educate other patients and relatives without cognitive problems about dementia

Table 2 List of knowledge and skills required by hospital staff in caring for persons with dementia as identified by participants in the focus groups 
